\title{
Encéphalite à parvovirus B19 de l'enfant
}

\author{
Childhood parvovirus B19 encephalitis \\ P. Meyer ${ }^{a, b, *}$, E. Jeziorski ${ }^{a}$, L. Bott-Gilton ${ }^{a}$, V. Foulongne ${ }^{c}$, F. Rivier $^{b}$, G. Rondoin ${ }^{d}$, \\ M. Rodière ${ }^{a}$ \\ a Service d'immuno-rhumatologie et maladies infectieuses pédiatriques, université \\ Montpellier-1, CHU de Montpellier, 34000 Montpellier, France \\ ${ }^{\mathrm{b}}$ Service de neuropédiatrie, université Montpellier-1, CHU de Montpellier, 34000 Montpellier, \\ France \\ ${ }^{\mathrm{C}}$ Laboratoire de virologie, université Montpellier-1, CHU de Montpellier, 34000 Montpellier, \\ France \\ dUnité de neurophysiologie clinique, département de neurologie, université Montpellier-1, \\ $\mathrm{CHU}$ de Montpellier, 34000 Montpellier, France
}

\section{Summary}

Introduction. Human parvovirus B19 (PVB19) causes erythema infectiosum or $5^{\text {th }}$ disease in childhood, which mainly affects children between 3 and 15 years of age. PVB19 infections have also been described in association with a variety of neurologic manifestations including encephalitis.

Case report. This 3-year 8-month-old boy developed febrile encephalitis (mental status change with seizures and left limb hypertonia) associated with a rash. The electroencephalographs revealed focal slowing with some spikes in front of the left centro-temporo-occipital areas ; bacteriological and biochemical cerebrospinal fluid (CSF) analysis were normal, brain radiologic studies (tomography and magnetic resonance imaging) were normal. The diagnosis of encephalitis associated with PVB19 primo infection was based on viral DNA detection in the serum and CSF using PCR and on the specific immunoglobulin M (without immunoglobulin G) detection in the serum.

Discussion. In France, encephalitis etiology is unknown in $48 \%$ of the cases. PVB19 accounts for $4.3 \%$ of undiagnosed meningoencephalitis in children. Although there is no specific sign, seizures and rash are reported in about one-half and one-quarter of cases, respectively.

Conclusion. Even if PVB19 research is not cited in the French or American infectious disease society recommendations on the diagnosis and management of infectious encephalitis, this virus may be responsible, especially in cases of child febrile rash. Therefore, PVB19 research seems reasonable if the clinical presentation is concordant in children due to its diagnostic simplicity and efficacy.

\footnotetext{
* Auteur correspondant.
}

e-mail : pagmeyer@yahoo.f

\section{Résumé}

Introduction. Le parvovirus B19 (PVB19) est l'agent responsable du mégalérythème épidémique ou $5^{\mathrm{e}}$ maladie, qui touche essentiellement l'enfant entre 3 et 15 ans. Même si elles sont rares, des manifestations neurologiques ont été décrites.

Cas clinique. Nous rapportons l'observation d'un patient de 3 ans et 8 mois qui avait présenté un tableau clinique d'encéphalite aiguë (troubles de la vigilance, hypertonie du membre supérieur droit, convulsions) dans un contexte d'éruption fébrile. L'électroencéphalogramme objectivait un ralentissement en regard des régions centrotemporo-occipitales gauches ; l'analyse cytobactériologique du liquide céphalorachidien (LCR) et l'imagerie cérébrale étaient normales. La sérologie pour le PVB19 était positive en immunoglobulines (Ig) M sans IgG en phase aiguë et les réactions de polymérisation en chaîne (PCR) spécifiques positives dans le sérum et le LCR avaient fait porter le diagnostic d'encéphalite à PVB19. Discussion. En France, $48 \%$ des encéphalites sont idiopathiques (Mailles et al., 2009). Chez l'enfant, le PVB19 serait responsable de 4,3\% de ces encéphalites sans agent causal identifié (Barah et al., 2001). Il n'y a pas de signe spécifique, néanmoins des convulsions et un rash peuvent être trouvés respectivement dans la moitié et un quart des cas. (Douvoyiannis et al., 2009).

Conclusion. Même si la recherche du PVB19 ne figure pas dans les recommandations de prise en charge diagnostique des encéphalites de la Société de pathologie infectieuse de langue française et de la Société nord-américaine de pathologie infectieuse, ce virus peut en être la cause notamment chez le grand enfant qui présente une éruption fébrile. Ainsi, en cas de contexte évocateur, il nous semble raisonnable d'effectuer la recherche du PVB19, agent pathogène « banal » de l'enfant, dont le diagnostic est simple et présente un bon rendement. 


\section{Introduction}

Le parvovirus B19 (PVB19), virus à acide désoxyribonucléique (ADN) simple brin non enveloppé, est l'agent infectieux responsable du mégalérythème épidémique, ou $5^{\mathrm{e}}$ maladie, qui fait partie des viroses exanthématique de l'enfant. L'éruption réalise un aspect souffleté des joues et un érythème figuré des membres qui touche préférentiellement les enfants entre 3 et 15 ans, la durée d'incubation est de 5 à 14 j. Dans la majorité des cas les patients sont asymptomatiques, mais de nombreuses autres manifestations ont été décrites au cours de la primo-infection : érythroblastopénie aiguë chez les patients atteints d'hémoglobinopathie, thrombopénie, agranulocytose, anasarque fœtoplacentaire, arthropathies chez l'adulte, myocardites, vascularites, artériopathie oblitérante et syndrome de fatigue chronique [1]. Parmi ces formes cliniques, des atteintes neurologiques ont été rapportées à type d'encéphalites, méningites, neuropathies périphériques, accidents vasculaires cérébraux, ataxies, myélites transverse, syndrome du canal carpien et syndrome de Guillain-Barré [2-5]. Nous rapportons un cas pédiatrique d'encéphalite à PVB19 d'évolution spontanée rapidement favorable.

\section{Observation}

Il s'agissait d'un patient de 3 ans et 8 mois issu de parents caucasiens non consanguins. Son développement psychomoteur était normal. Une semaine avant l'hospitalisation, il avait présenté une éruption cutanée papuleuse non prurigineuse étiquetée varicelle, spontanément résolutive en $5 \mathrm{j}$. Quarantehuit heures plus tard, il était trouvé somnolent avec une flexion hypertonique du membre supérieur droit (MSD), puis survenait une perte de contact avec révulsion oculaire et perte d'urines. L'enfant était alors adressé au service des urgences pédiatriques. II avait une fièvre à $38,4{ }^{\circ} \mathrm{C}$ et l'examen neurologique trouvait des troubles de la vigilance avec un score de Glasgow à 8 et une hypertonie persistante du MSD avec main en griffe. II existait un érythème papuleux non prurigineux d'un demi centimètre de diamètre sur le bras droit et le thorax. L'enfant bénéficiait alors d'une dose de charge de diazépam par voie intraveineuse (IV) $(0,5 \mathrm{mg} / \mathrm{kg})$ et un traitement probabiliste par acyclovir IV était débuté. L'évolution était rapidement favorable, l'état de conscience s'améliorant rapidement, le score de Glasgow revenant à 14 en quelques heures mais l'hypertonie du MSD régressant plus lentement, la normalisation totale de l'examen clinique étant obtenue en $48 \mathrm{~h}$. L'enfant était apyrétique dès la première prise de paracétamol. Les principaux examens complémentaires sont présentés dans le tableau I.

La ponction lombaire effectuée le premier jour ramenait un liquide acellulaire dont l'analyse biochimique était normale. La recherche de virus de l'herpès dans le LCR par PCR (polymerase chain reaction) était négative et l'interféron $\alpha$ était inférieur à $2 \mathrm{UI} / \mathrm{mL}$, permettant l'arrêt de l'acyclovir au bout de $48 \mathrm{~h}$. En l'absence de récidive des convulsions, le diazépam était relayé par du valproate de sodium par voie orale. L'électroencéphalogramme (EEG) réalisé au $5^{\mathrm{e}} \mathrm{j}$ objectivait la persistance d'un ralentissement modéré en regard des régions centro-temporo-occipitales gauches (fig. 1). L'enfant sortait au $6^{\mathrm{e}} \mathrm{j}$ sous traitement anticomitial. L'examen clinique et l'EEG de veille et de sommeil réalisés 1 mois plus tard étaient strictement normaux. Une sérologie pour le PVB19 réalisée au même moment n'a malheureusement pas pu être interprétée pour des raisons techniques. Sur le plan étiologique, la sérologie étant positive en IgM et négative en IgG, la PCR étant positive dans le sérum et le LCR, et aucun autre agent viral n'ayant été trouvé, nous avons retenu le diagnostic d'encéphalite à PVB19.

\section{Tableau I}

\section{Résultats des examens complémentaires.}

Numération formule sanguine, ionogramme sanguin, bilan hépatique, CRP, procalcitonine : normaux

Sérologies Borrelia burgdorferi, Bartonella henselae, Brucella, Chlamydiae, Mycoplasma pneumoniae, Rickettsia conori : négatives Sérologies VIH, EBV, CMV, HTLV-1 : négatives

Sérologies rubéole, rougeole, oreillons : immunité ancienne

Sérologie parvovirus B19 : infection récente (positive en IgM, négative en IgG)

PCR parvovirus B19 dans le sang : positive

Analyse du LCR

Glycorachie, chlorurorachie et protéinorachie : normales

Éléments blancs et rouges $<10 / \mathrm{mm}^{3}$

$\mathrm{PCR}$ parvovirus $\mathrm{B} 19$ : positive

PCR CMV, adénovirus, HSV, entérovirus, EBV, VZV, HHV6 : négatives

Dosage de l'interféron $\alpha<2 \mathrm{UI} / \mathrm{mL}$

Tomodensitométrie cérébrale avec injection de produit de contraste : normale IRM cérébrale avec injection de gadolinium : normale

$P C R$ : réaction de polymérisation en chaîne; $L C R$ : liquide céphalorachidien; $E E G$ : électroencéphalogramme; IRM : imagerie par résonance magnétique; VIH : virus d'immunodéficience humaine; VZV: varicelle zona virus; HHV6 : human herpes virus type 6 ; CMV : cytomagalovirus; EBV : Epstein-Barr virus; HSV : virus herpès simplex; CRP : protéine C réactive ; HTLV: human $T$ cell leukaemia virus. 


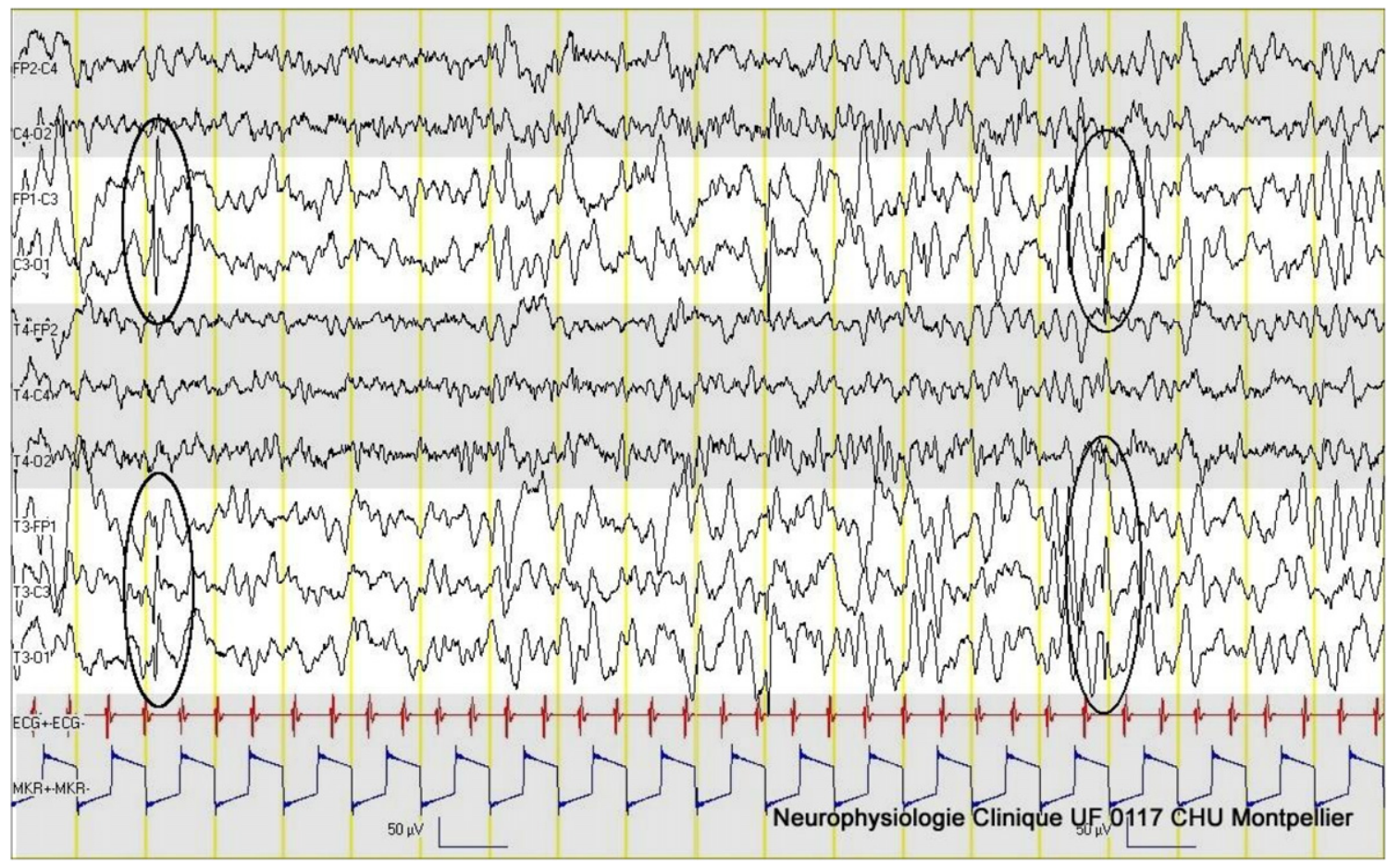

Figure 1. Electroencéphalogramme au cinquième jour. Ralentissement hémisphérique gauche (bandes claires) surchargé de quelques pointes (entourées).

\section{Discussion}

Le PVB19 est un des principaux représentants de la famille des parvoviridae, qui comprend notamment le virus adéno-associé (AAV), le bocavirus humain et le Parv4. Le court génome de ces virus ne code pour aucune ADN-polymérase, ce qui leur impose d'infecter des cellules en réplication (phase $S$ ) ou de co-infecter avec un autre virus pour une réplication efficace de leur ADN [6]. De ce fait, les AAV présentent un fort potentiel thérapeutique comme vecteurs de gènes dans un but de thérapie génique [7]. L'infection à PVB19 survient le plus souvent dans l'enfance, et environ $60 \%$ des grands enfants et des adultes sont séropositifs pour le PVB19 (contre 5 à $15 \%$ des enfants de moins de 5 ans) [8]. Les manifestations neurologiques lors de la primo-infection à PVB19 sont rares et surviennent majoritairement chez les enfants [3].

La première description d'encéphalite associée à la $5^{\mathrm{e}}$ maladie remonte à 1970 et concernait un garçon de 8 ans [9]. Dans une revue de la littérature, Douvoyiannis et al. ont recensé 39 encéphalites, encéphalopathies et méningoencéphalites parmi 81 patients ayant présenté une manifestation neurologique associée à une infection à PVB19 entre 1970 et juillet 2008 . Parmi ces 39 patients, 30 (77\%) étaient des enfants, l'âge médian étant de 9 ans ( 2 mois à 68 ans) et $64 \%$ étaient immunocompétents. La drépanocytose représentait plus de la moitié des causes d'immunodépression [3].

Sur le plan clinique, l'enfant de notre observation avait présenté un tableau initial de troubles de la vigilance avec des convulsions associés à une éruption cutanée. Cela correspond aux cas rapportés dans la littérature : des troubles de la vigilance sont présents dans $49 \%$ des cas (le coma dans $15 \%$ des cas), des convulsions le plus souvent généralisées dans $46 \%$ des cas (avec état de mal dans $10 \%$ des cas), un signe neurologique focal dans $28 \%$ des cas et un rash cutané dans $26 \%$ des cas. Notre patient n'avait pas présenté les autres signes cliniques décrits : syndrome grippal (40\%), raideur méningée (5\%), hallucinations auditives et visuelles (10 \%), ataxie (8 \%) et arthralgies (8 \%) [3]. Les symptômes grippaux et le rash cutané sont plus fréquemment observés chez les patients immunocompétents.

Sur le plan paraclinique, l'analyse cytochimique du LCR était normale dans notre observation : dans la série de Douvoyiannis et al., l'analyse biochimique et cytobactériologique du LCR était normale dans respectivement $33 \%$ et $52 \%$ des cas. En revanche, la détection de l'ADN du PVB19 par PCR était positive dans le sang et le LCR de notre patient, ce qui a été trouvé dans respectivement $81 \%$ et $85 \%$ des cas (le diagnostic étant posé sur la sérologie dans le sang ou le LCR en cas de négativité) [3]. Tous germes confondus, l'analyse cytologique et bactériologique du LCR est normale dans $10 \%$ des cas d'encéphalites [10].

L'imagerie par résonance magnétique (IRM) cérébrale réalisée chez notre patient était normale, comme dans $37 \%$ des cas rapportés. Lorsqu'elles sont présentes, les anomalies sont variées en termes de localisation, pouvant intéresser la substance blanche ou la substance grise. Des anomalies à la 
tomodensitométrie cérébrale sont détectées dans $46 \%$ des cas [3].

L'évolution de la maladie s'était révélée très rapidement favorable chez notre patient, avec normalisation de l'examen clinique en $48 \mathrm{~h}$ et EEG normal à 1 mois sous valproate de sodium. Douvoyiannis et al. avaient rapporté une durée moyenne de la maladie plus longue $(55,7 \mathrm{j}$; extrêmes : 3 à $198 \mathrm{j}$ ) et un pronostic défavorable avec des séquelles neurologiques ou des décès dans $31 \%$ des cas [3]. Il est à noter que la détection de l'ADN du PVB19 par PCR dans le LCR ne semble pas être un facteur de mauvais pronostique, tendance que confirme notre observation.

Sur le plan thérapeutique, notre patient n'a reçu que des benzodiazépines puis du valproate de sodium à visée anticonvulsivante. Les thérapeutiques immunomodulatrices (corticothérapie ou immunoglobulines IV) ont été proposées dans les (méningo-)encéphalites à PVB19 de l'enfant comme de l'adulte, sans efficacité prouvée, avec même un effet délétère dans certaines observations [3,4]. L'absence d'étude contrôlée ne permet pas de conclure de manière formelle quant à l'intérêt de ces thérapeutiques dans cette indication.

En France, une étude prospective multicentrique réalisée en 2007 avait recensé $48 \%$ d'encéphalites idiopathiques [11]. Par ailleurs, une étude rétrospective portant sur 162 enfants ayant présenté une encéphalite, une méningoencéphalite ou une encéphalopathie sans cause initialement mise en évidence, avait détecté l'ADN du parvovirus B19 par PCR dans le LCR dans $4,3 \%$ des cas [12] ( $4,7 \%$ dans une série adulte et pédiatrique [13]).

La physiopathologie de l'atteinte du système nerveux central par le PVB19 reste inconnue : le PVB19 a été retrouvé au niveau du cortex préfrontal dorsolatéral dans $14 \%$ des échantillons d'une série de 104 cerveaux issus de la Stanley Medical Research Institute Brain Collection [14] et plusieurs mécanismes ont été proposés pour expliquer la pathogénicité du virus chez seulement quelques individus : toxicité directe, dysrégulation immunitaire avec relargage de cytokines dans le LCR, dépôts de complexes immuns dans les cellules endothéliales et accumulation intracellulaire de protéine NS1 [5].

En 2006, la Société de pathologie infectieuse de langue française (SPILF) a publié des recommandations de bonnes pratiques diagnostiques pour les encéphalites infectieuses de l'adulte et de l'enfant non immunodéprimé [15]. La priorité est de rechercher en $1^{\text {re }}$ intention les causes curables, souvent orientées par la clinique et l'analyse cytobactériologique et biochimique du LCR : HSV (virus herpès simplex) 1 et 2, listeria, M. tuberculosis, P. falciparum, borrelia, syphilis, brucellose. En l'absence d'orientation étiologique, la SPILF recommande des recherches en 3 niveaux réalisées sur 2 prélèvements de LCR et indique les agents à rechercher tout particulièrement en cas de rash cutané (tableau II). La recherche de PVB19 n'est pas citée dans ces recommandations. En 2008, la Société de pathologie infectieuse nord-américaine a publié des recommandations pour le diagnostic et la prise en charge des encéphalites infectieuses de l'adulte et de l'enfant [10] (tableau III). Comme précédemment, le PVB19 n'est pas cité. Notre observation et les données de la littérature montrent que le PVB19 est un agent non exceptionnel d'encéphalite. L'enquête étiologique d'une encéphalite est une démarche souvent difficile mais indispensable car la découverte d'un agent infectieux responsable, même si elle n'aboutit que rarement à un traitement spécifique, permet de prendre des mesures prophylactiques et surtout d'écarter d'autres diagnostics. Ainsi, le PVB19 devrait être recherché en cas de

Tableau II

Recherches infectieuses recommandées par la Société de pathologie infectieuse de langue française (SPILF) en cas de suspicion d'encéphalite (en l'absence de circonstances épidémiologiques particulières) [15].

\begin{tabular}{|c|c|c|c|}
\hline & $1^{e r}$ niveau & $\begin{array}{l}2^{\mathrm{e}} \text { niveau (sur } 2^{\mathrm{e}} \text { PL en cas } \\
\text { de négativité du } 1^{\text {er }} \text { niveau) }\end{array}$ & $\begin{array}{l}3^{e} \text { niveau (sur échantillon de LCR } \\
\text { de la } 2^{\mathrm{e}} \text { PL conservé au préalable) }\end{array}$ \\
\hline Agents viraux & $\begin{array}{l}\text { Sérologie VIH } \\
\text { Étude du LCR } \\
\text { PCR HSV } 1 \text { et } 2 \\
\text { PCR VZV }\end{array}$ & $\begin{array}{l}\text { Étude du LCR } \\
2^{\mathrm{e}} \text { PCR HSV } \\
\text { PCR entérovirus } \\
\text { PCR CMV } \\
\text { PCR EBV } \\
\text { PCR adénovirus } \\
\text { PCR HHV6 }\end{array}$ & $\begin{array}{l}\text { Étude du LCR } \\
\text { Influenza A et B } \\
\text { Parainfluenzae } \\
\text { Rougeole } \\
\text { Oreillons } \\
\text { Rubéole } \\
\text { West Nile } \\
\text { Toscana virus } \\
\text { Virus JC } \\
\text { Parechovirus } \\
\text { LCMV }\end{array}$ \\
\hline Agents bactériens & M. pneumoniae & $\begin{array}{l}\text { Chlamydia sp } \\
\text { Borrelia burgdorferi } \\
\text { Coxiella burnetti } \\
\text { Bartonella hensellae }\end{array}$ & $\begin{array}{l}\text { Rickettsiae et apparentés } \\
\text { Tropheryma whipplei } \\
\text { Ehrlichia schaffensii }\end{array}$ \\
\hline
\end{tabular}

En cas de rash cutané : rechercher en particulier rougeole, rubéole, VZV, borrelia, enterovirus, M pneumoniae. PL : ponction lombaire; LCR : liquide céphalorachidien, PCR : réaction de polymérisation en chaîne; VIH : virus d'immunodéficience humaine; VZV : varicelle zona virus; HHV6 : human herpes virus type 6 ; CMV : cytomagalovirus ; EBV : Epstein-Barr virus; HSV : virus herpès simplex; LCMV : lymphocytic choriomeningitis virus ; 
Tableau III

Explorations recommandées en première intention par la Société de pathologie infectieuse nord-américaine en cas de suspicion d'encéphalite [10].

Culture virale des sécrétions nasopharyngées et des selles

Recherche des principaux virus respiratoires dans les sécrétions nasopharyngées

PCR M. pneumoniae dans les sécrétions nasopharyngées

Sérologie VIH chez le séronégatif (charge virale en cas de forte suspicion)

Sérologie Epstein-Barr virus

Sérologie M. pneumoniae

Sérologies en fonction de l'origine géographique et de la période : West Nile virus, St. Louis encephalitis virus, Eastern equine encephalitis virus, Venezuelan equine encephalitis virus, La Crosse virus

Étude du LCR

Recherche d'IgM spécifiques en fonction de l'origine géographique et de la période : West Nile virus, St. Louis encephalitis virus IgM varicelle zona virus

PCR herpes simplex virus 1, herpes simplex virus 2, varicelle-zona virus, Epstein-Barr virus et entérovirus

Recherches bactériennes à envisager : Rickettsiea et apparentés, spirochètes, mycobactéries

En cas de rash cutané : rechercher en particulier le VZV, HHV6, la rubéole, les entérovirus, le VIH, Rickettsia rickettsii, M. pneumoniae, Borrelia burgdorferi, Treponema pallidum et, de manière beaucoup plus rare dans nos contrées, le virus B (ceercopithecine herpesvirus), Ehrlichia chaffeensis et Anaplasma phagocytophilum. LCR : liquide céphalorachidien ; PCR : réaction de polymérisation en chaîne; VIH: virus d'immunodéficience humaine; VZV : varicelle zona virus; HHV6 : human herpes virus type 6 ; IgM : immunoglobuline monoclonale.

contexte clinique évocateur chez l'enfant, et ce d'autant que des moyens diagnostiques simples sont disponibles dans de nombreux centre hospitaliers.

\section{Conclusion}

En France, $48 \%$ des encéphalites sont idiopathiques [11]. Le PVB19 est responsable chez l'enfant de $4,3 \%$ d'entre elles lorsqu'aucun agent causal n'a été identifié [12]. Les recommandations françaises de 2006 et nord-américaines de 2008 pour le diagnostic étiologique des encéphalites infectieuses omettent de citer ce virus qui n'est pourtant pas une cause exceptionnelle d'infection aiguë du système nerveux central [10]. À travers cette observation, nous posons la question de l'intérêt d'une recherche du PVB19 dans le sang et le LCR en cas d'encéphalite sans cause évidente chez l'enfant.

\section{Déclaration d'intérêts}

Les auteurs déclarent ne pas avoir de conflits d'intérêts en relation avec cet article.

\section{Remerciements}

Les auteurs tiennent à remercier Mlle Virginie Morin pour son aide précieuse dans la rédaction de cet article.

\section{Références}

[1] Young NS, Brown KE. Parvovirus B19. N Engl J Med 2004;350: 586-97.
[2] Minohara Y, Koitabashi Y, Kato T, et al. A case of Guillain-Barré syndrome associated with human parvovirus B19 infection. J Infect 1998;36:327-8.

[3] Douvoyiannis M, Litman N, Goldman DL. Neurologic manifestations associated with parvovirus B19 infection. Clin Infect Dis 2009;48:1713-23.

[4] Greco F, Barbagallo ML, Chiodo DC, et al. Severe ataxia as a complication of human parvovirus B19 acute encephalitis in a child. J Child Neurol 2008;23:1078-80.

[5] Barah F, Vallely PJ, Cleator GM, et al. Neurological manifestations of human parvovirus B19 infection. Rev Med Virol 2003;13:185-99.

[6] Brown KE. The expanding range of parvoviruses which infect humans. Rev Med Virol 2010;20:231-44.

[7] Heilbronn R, Weger S. Viral vectors for gene transfer: current status of gene therapeutics. Handb Exp Pharmacol 2010;197: 143-70.

[8] Cohen BJ, Buckley MM. The prevalence of antibody to human parvovirus B19 in England and Wales. J Med Microbiol 1988; 25:151-3.

[9] Balfour HH, Schiff GM, Bloom JE. Encephalitis associated with erythema infectiosum. J Pediatr 1970;77:133-6.

[10] Tunkel AR, Glaser CA, Bloch KC, et al. The management of encephalitis: clinical practice guidelines by the infectious diseases society of America. Clin Infect Dis 2008;47:303-27.

[11] Mailles A, Stahl J. Infectious encephalitis in France in 2007: a national prospective study. Clin Infect Dis 2009;49:1838-47.

[12] Barah F, Vallely PJ, Chiswick ML, et al. Association of human parvovirus B19 infection with acute meningoencephalitis. Lancet 2001;358:729-30.

[13] Haseyama K, Kudoh T, Yoto $Y$, et al. Detection of human parvovirus B19 DNA in cerebrospinal fluid. Pediatr Infect Dis J 1997;16:324-6.

[14] Hobbs JA. Detection of adeno-associated virus 2 and parvovirus B19 in the human dorsolateral prefrontal cortex. J Neurovirol 2006;12:190-9.

[15] SPILF. Pour une bonne pratique de la prise en charge des encéphalites en France. Paris, France : Société de pathologie infectieuse de langue française. Disponible sur internet: URL: http://www.infectiologie.com/site/medias/_documents/ 2006-encephalites.pdf. Page web consultée le 15 janvier 2011. 